\title{
Reduction of EMI by Combining Interleaving and Modulation Techniques in Multiconverter Topology
}

\author{
J. Mon, J. Gago, D. González, J. Balcells, R. Fernández, I. Gil \\ Universitat Politècnica de Catalunya (Terrassa, Spain) \\ jmon@eel.upc.edu
}

\begin{abstract}
In this paper an evaluation of different modulations techniques that combine switching frequency modulation and interleaving is presented. The objective of these modulations is to cancel certain harmonics of EMI and to reduce the amplitude of the remaining harmonics. A four channel parallel buck converter has been used in order to evaluate the modulations proposed. According experimental results, significant reduction of EMI is possible by combining both techniques instead of using them separately.
\end{abstract}

Index Terms- Electromagnetic Compatibility (EMC), Electromagnetic Interference (EMI), Switching Frequency Modulation (SFM), power electronic, power converters.

\section{INTRODUCTION}

In recent years, the switching frequency modulation has been explored, as EMI reduction technique, in switching mode power supplies (SMPS) with single converter topology. However, the use of this technique in distributed or modular power systems made up of several power converters has not been studied. The switching mode power supplies generate signals with high $d v / d t$ and $d i / d t$. This situation produces electromagnetic interference (EMI) in the power lines that can affect other circuits or devices. The SMPS normally are designed to operate with constant switching frequency and variable duty cycle $(D)$, which is adjusted according to the response of the control loop. Therefore, the main component of the EMI is the switching frequency and its harmonics.

Traditionally the techniques used in order to reduce EMI in power converters are based in EMI filters, snubber network, soft switching, etc. However, in nineties years, a new technique called switching frequency modulation (SFM) [1,2] has appeared with the aim of reducing EMI in SMPS. This technique consists of modulating in frequency the switching pattern of power converters. In the last years, several works have explored different aspects of this technique in centralized power systems [3-7]. However, there is another aspect to study, that is, the use of SFM in distributed or modular power systems.

On the other hand, in modular power systems with parallel topology, interleaving technique is commonly used to reduce EMI and output voltage ripple. These system topologies are used in applications that require high level of power or redundancy, such as microprocessors power supplies. The interleaving technique consists of introducing a given shift delay among the switching patterns of the converters, resulting in harmonics cancellation effect [8-12].
In this work, different modulations techniques in combination with interleaving that can be applied in modular power supplies systems with parallel topology are presented.

The paper is organized as follows. First of all, in section II some theoretical considerations regarding SFM and interleaving techniques are presented. In section III, two different modulations that combine the interleaving and SFM techniques are analysed and discussed. In section IV, the practical setup implemented is described whereas the obtained experimental results are presented in section $\mathrm{V}$, paying special attention to the EMI reduction when we use the proposed modulation techniques. Finally, in section VI the main conclusions are summarized and some future works are pointed out.

\section{THEORETICAL CONSIDERATIONS}

\section{A. Switching Frequency Modulation}

The switching frequency modulation technique mainly consist of varying the switching frequency around a central value, $f_{c}$, according to a modulation profile, $V_{m}(t)$.

Depending on the modulation profile, there are two possibilities to modulate the switching frequency:

- Random Modulation: The switching frequency is modulated by a random profile with a known probability density function.

- Periodic Modulation: The switching frequency is modulated by a periodic profile which amplitude defines the maximum frequency deviation $\left(\Delta f_{c}\right)$.

In this study, the periodic modulation case has been selected because it is easy to predict the switching pattern. A deeper description of the differences between random and periodic modulation can be found in [3].

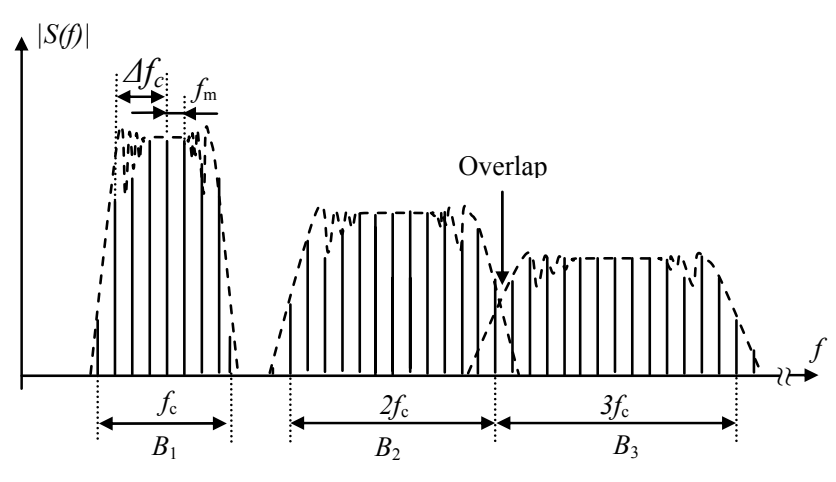

Fig. 1. Spread bands of harmonics in SFM. 


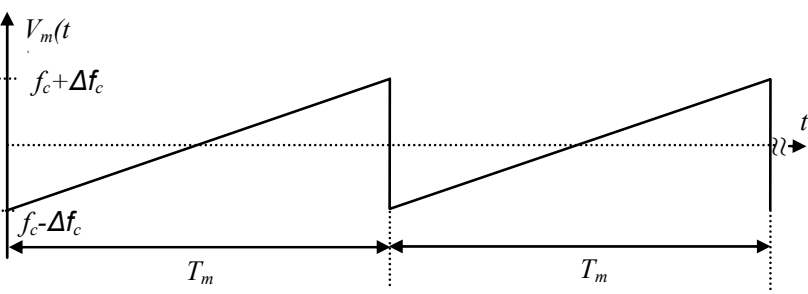

(a)

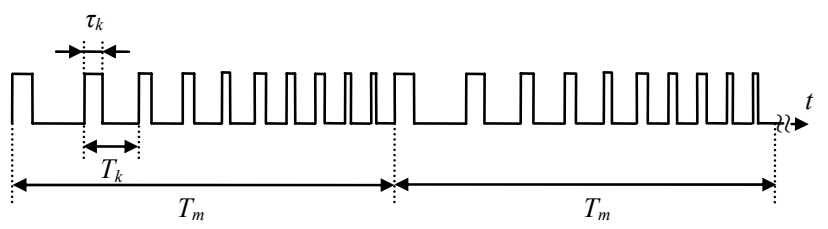

(b)

Fig. 2. Switching frequency modulation. (a) Periodic modulation profile. (b) Frequency modulated switching pattern.

In case of using a periodic modulation profile the energy of the interference harmonics is distributed in side-bands that are generated by modulation process (Fig. 1). The distance between two consecutive side-bands harmonics is given by the frequency of modulation profile $\left(f_{m}\right)$.

The EMI reduction with the SFM technique when periodic profile is used, it depends on the modulation index $\left(m_{f}\right)$. The relationship between $m_{f}$ and other modulation parameters are shown in (1) [4].

$$
m_{f}=\frac{\Delta f_{c}}{f_{m}}
$$

By increasing $m_{f}$, the amplitude of electromagnetic interference harmonics is reduced. However, some considerations that limit the maximum value of $\Delta f_{c}$ and minimum value of $f_{m}$ must be taken into account. The maximum frequency deviation depends of $f_{c}$, a high value of $\Delta f_{c}$ can originate an overlap effect between consecutive side-bands (Fig. 1). The resolution bandwidth of spectrum analyzer (RBW) limits the minimum value of $f_{m}$. In fact, it is important to satisfy the condition $f_{m}>\mathrm{RBW}[1,3]$. On the other hand, the RBW value is imposed by the EMI directives.

Another important parameter is the modulation profile, $V_{m}(t)$, (Fig.2a) which defines the envelop EMI spectrum [4]. From the point of view of the electromagnetic interferences, the most important modulation profile is sawtooth or triangular [4-5]. Figure $2 \mathrm{~b}$ shows the resulting switching pattern when we use a sawtooth modulation profile.

\section{B. Interleaving}

In modular power supplies with parallel topology the interleaving technique is used to equally share the total power to be delivered among the number of converters, $N$ [8]. In this case all converters operate with the same frequency but a shift delay equal to $T_{c} / N$ is introduced in the switching pattern of each converter (Fig.3a), where $T_{c}$ is the switching period. From

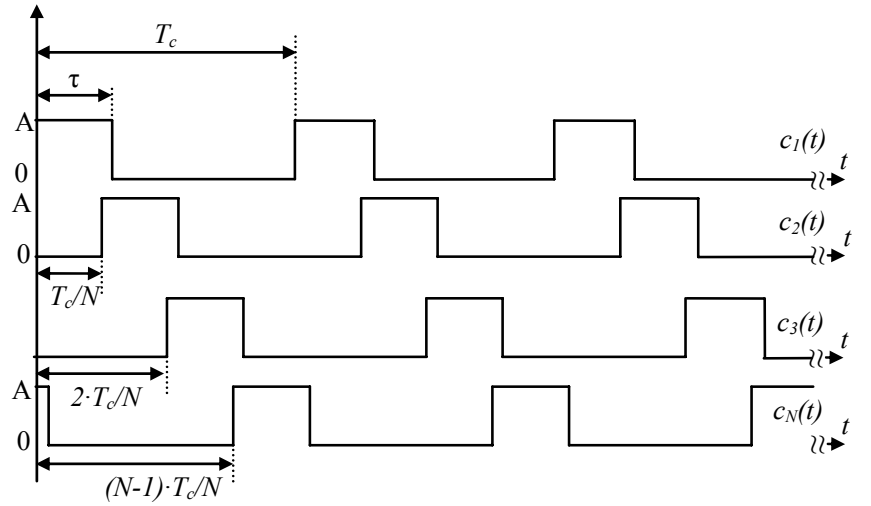

(a)

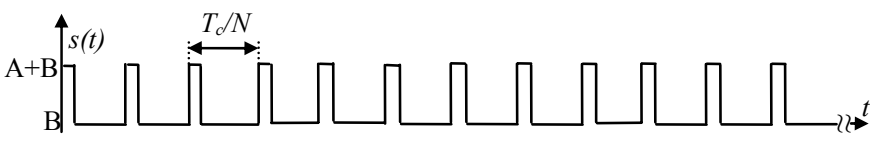

(b)

Fig. 3. Interleaving technique for $N=4$. (a) Switching pattern. (b) Equivalent source of noise pattern.

the point of view of the noise generation, the combination of these $N$ switching pattern $\left(c_{i}(t)\right.$, with $\left.i=1,2, \ldots N\right)$ results in a signal $s(t)$, which correspond to the equivalent noise generation pattern (Fig. 3b).

The expressions in time domain of $c_{i}(t)$ and the equivalent noise generation pattern, $s(t)$ is given by (2), where subindex $i$ corresponds to each of the $N$ switching patterns and $k$ notes the switching cycle.

$$
\begin{aligned}
& s(t)=\sum_{i=1}^{N} c_{i}(t)=A \cdot\left[B+\sum_{k=0}^{\infty}\left(u\left(t-k \frac{T_{c}}{N}\right)-u\left(t-k \frac{T_{c}}{N}-\left(\tau-\frac{T_{c}}{N}\right)\right)\right)\right] \\
& c_{i}(t)=A \cdot \sum_{k=0}^{\infty}\left(u\left(t-k T_{c}-\frac{T_{c}}{N}(i-1)\right)-u\left(t-k T_{c}-\tau-\frac{T_{c}}{N}(i-1)\right)\right) \\
& B=\left\{\begin{array}{lc}
0, & \tau<\frac{T_{c}}{N} \\
1, & \frac{T_{c}}{N} \leq \tau<\frac{2 \cdot T_{c}}{N} \\
\cdots & (N-1) T_{c} \\
N-1, & \frac{\left(N<T_{c}\right.}{N}
\end{array}\right.
\end{aligned}
$$

The equivalent noise source pattern generated by all converters is equal to the generated by a single converter switching at a constant frequency of $N \cdot f_{c}$. Whereas in the switching pattern of each individual converter harmonic appears at $f_{c}$ (Fig.4a), on the equivalent source of noise pattern they only appear at $N \cdot f_{c}$ (Fig.4b). Thus, the interleaving technique has shown itself to be a harmonic cancellation method. The relationship in frequency domain between $C_{i}(w)$ and $S(w)$ is demonstrated in [12] and is given by (3), where $n$ is the harmonic order and $w_{c}$ is $2 \cdot \pi \cdot f_{c}$. 


$$
\begin{aligned}
|S(w)|= & \left|F\left\{\sum_{i=1}^{N} C_{i}(t)\right\}\right|=\left|C_{1}(w)\right||M(w)| \\
M(w)= & \frac{1-e^{-j \frac{n \cdot w_{c} T_{c} \cdot N}{N}}}{1-e^{-j \cdot \frac{n \cdot w_{c} \cdot T_{C}}{N}}}=\frac{1-e^{-j \cdot 2 \cdot \pi \cdot n}}{1-e^{-j \cdot 2 \cdot \pi \cdot n}}=\left\{\begin{array}{cc}
0 & n \neq p \cdot N \\
N & n=p \cdot N
\end{array} \quad p=0,1,2,3, \ldots\right.
\end{aligned}
$$

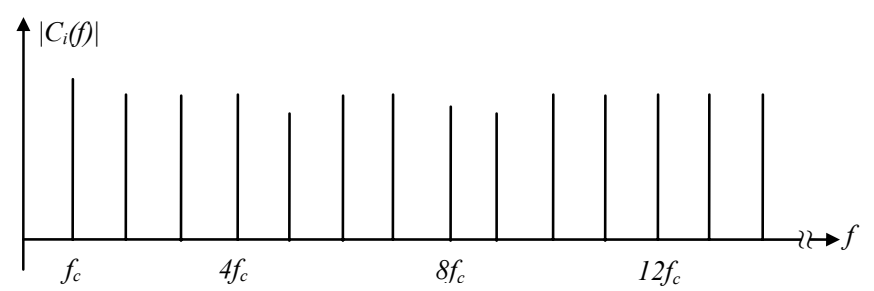

(a)

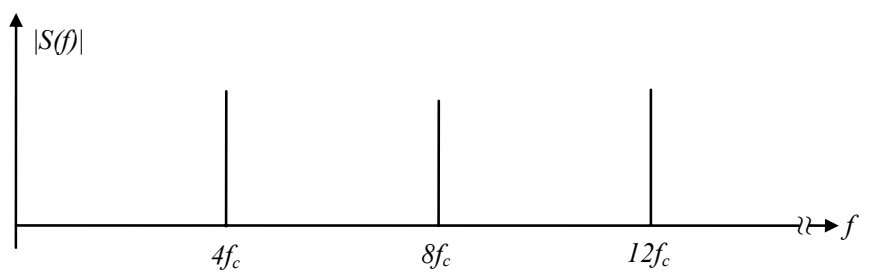

(b)

Fig. 4. Harmonics cancellation achieved with interleaving for $N=4$. (a) Switching pattern spectrum. (b) Equivalent source of noise pattern spectrum.

\section{ANALYSIS OF MODULATION TECHNIQUES IN PARALLEL MULTICONVERTER TOPOLOGY}

There are several possibilities of combined SFM techniques (Fig. 2) and interleaving (Fig. 3). In this section two of them are analyzed: the switching frequency modulation with constant shift delay and the switching frequency modulation with variable delay.

A. Switching Frequency Modulation with constant shift delay.

The switching frequency modulations with constant delay (CDFM) consist of modulating in frequency each switching pattern and introducing a constant shift delay. The delay is a multiple of the period switching pattern $\left(T_{m}\right)$ divided by the numbers of the converters $(N)$. Figure 5 shows the switching pattern for $N$ converters when this technique is applied. As is observed, the procedure is similar to interleaving techniques (Fig. 3), but in this case the switching patterns have a variable frequency according to the modulation profile (Fig.2).

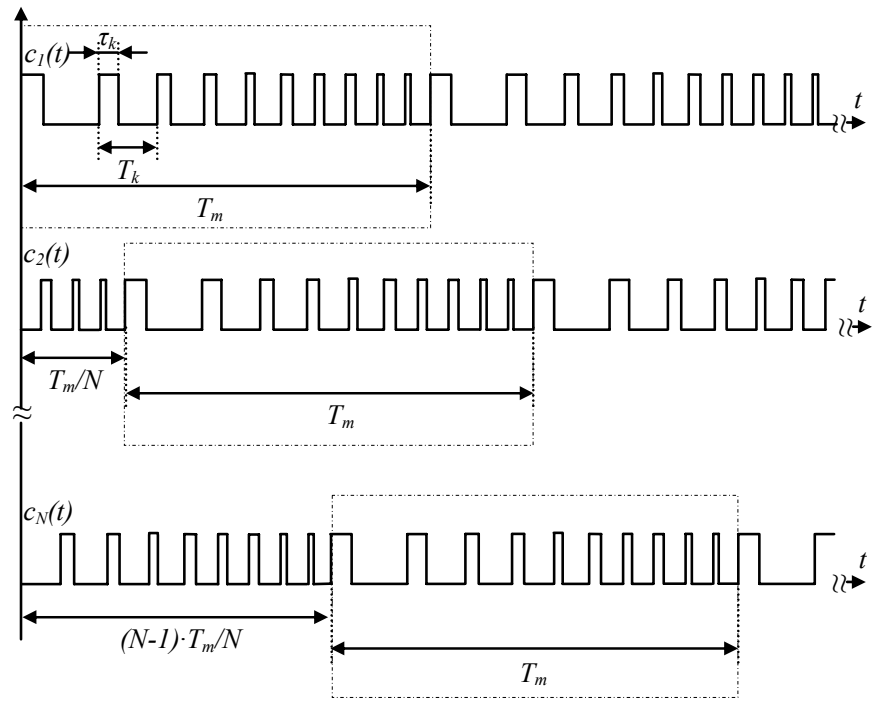

Fig. 5 Switching pattern of $N$ converters if CDFM technique is applied.

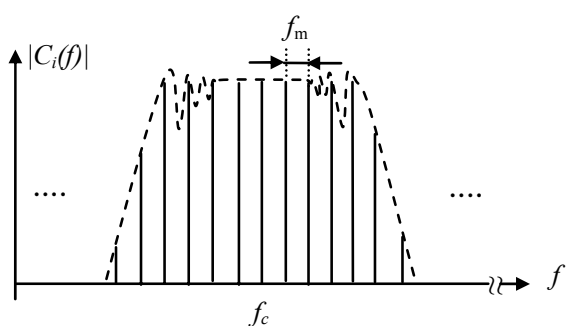

(a)

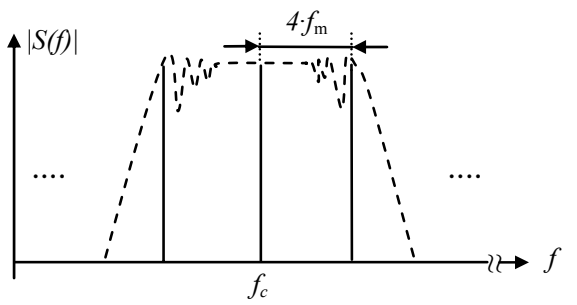

(b)

Fig. 6. Frequency spectrum for $N=4$. (a) Switching pattern. (b) Equivalent source of noise pattern for CDFM.

From (3) is it possible to obtain the equivalent noise spectrum of CDFM technique (4), just replacing, $w_{c}$ and $T_{c}$ by $w_{m}$ and $T_{m}$, respectively.

$$
\begin{aligned}
& |S(w)|=\left|F\left\{\sum_{i=1}^{N} C_{i}(t)\right\}\right|=\left|C_{1}(w)\right||M(w)| \\
& M(w)=\frac{1-e^{-j \frac{n \cdot w_{m} T_{m} \cdot N}{N}}}{1-e^{-j \cdot \frac{n \cdot w_{m} \cdot T_{m}}{N}}}=\frac{1-e^{-j \cdot 2 \cdot \pi \cdot n}}{1-e^{-j \cdot \frac{2 \cdot \pi \cdot n}{N}}}=\left\{\begin{array}{ll}
0 & n \neq p \cdot N \\
N & n=p \cdot N
\end{array} \quad p=0,1,2,3, \ldots\right.
\end{aligned}
$$


In Figure 6 the switching pattern spectrum (Fig.6a) and the switching pattern with constant delay spectrum (Fig.6b) are compared. As is observed, if the constant delay is introduced, only harmonics at $N \cdot f_{m}$ appears. Therefore, it is feasible to increase $m_{f}$ by decreasing $f_{m}(1)$, due to the minimum value of $f_{m}$ have to be $N$ times lower (5). As a result, it is possible to increase the EMI reduction when switching frequency modulation with constant shift delay technique is used.

$$
f_{m}>\frac{R B W}{N}
$$

\section{B. Switching Frequency Modulation with variable delay}

The switching frequency modulations with variable delay (VDFM) consist of applying interleaving in each switching cycle and at the same time using a frequency modulation technique. Figure $7 \mathrm{a}$ shows the switching pattern for 4 converters when VDFM are applied $(N=4)$. As is observed, a variable delay of pulse position, $\varepsilon_{k}$, is introduced in each switching period, in order to apply the interleaving technique in each switching cycle. In the first switching pattern $\varepsilon_{k}$ is constant and equal to 0 , but in the next switching patterns it varies depending on the instantaneous switching period $\left(T_{k}\right)$.

The relationship between periods are shown in (6), where $L$ is the number of switching cycles contained in a period of the modulation profile and must be an integer.

$$
f_{c}=\frac{1}{T_{c}} \quad ; \quad T_{c}=\frac{1}{L} \cdot \sum_{k=1}^{L} T_{k} \quad ; \quad L=\frac{T_{m}}{T_{c}}
$$

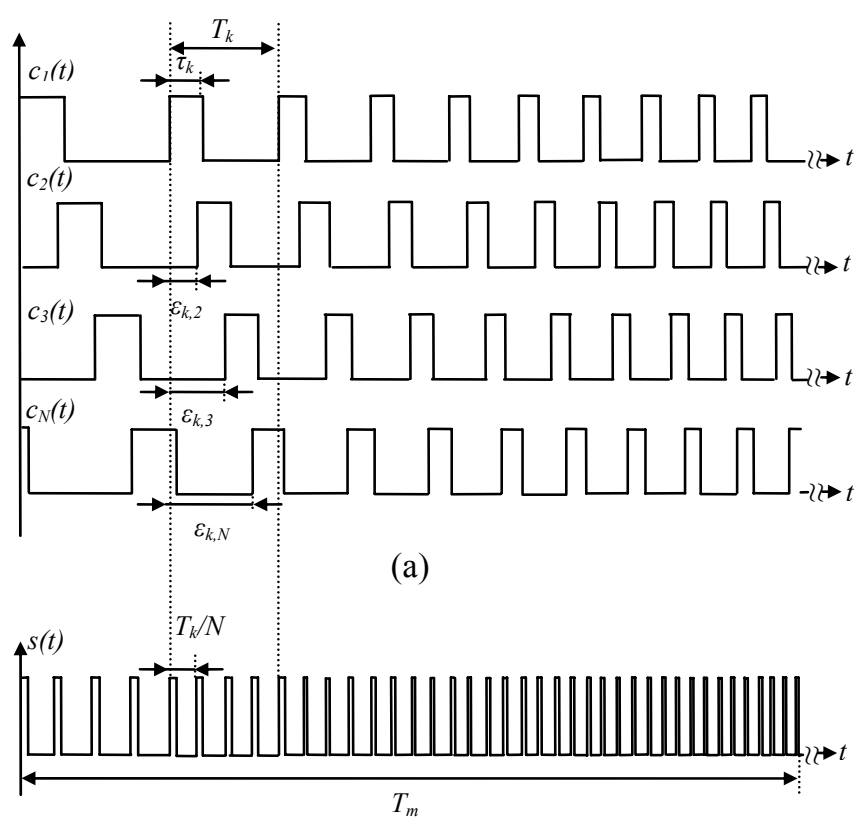

(b)

Fig. 7. Switching pattern if VDFM technique is applied for $N=4$. (a) Switching pattern. (b) Equivalent source of noise pattern.
The delay of pulse position inside a switching period is given by (7) where $i$ identifies the converter $(i=1,2,3,4, \ldots, N)$ and the equivalent noise source for 4 converters using this techniques is show on Figure $7 \mathrm{~b}$.

$$
\varepsilon_{k, i}=\frac{T_{k}}{N} \cdot(i-1)
$$

From Figure $7 \mathrm{~b}$ can be observed that the equivalent noise source, $s(t)$, is similar to switching frequency modulated pattern (Fig.2b) with central frequency of $N \cdot f_{c}$. In Figure 8 , the switching pattern spectrum (Fig.8a) and the equivalent noise spectrum for VDFM techniques (Fig.8b) are compared. It is observed a strong reduction on EMI when VDFM is applied, because only harmonic around $4 \cdot f_{c}$ appears.

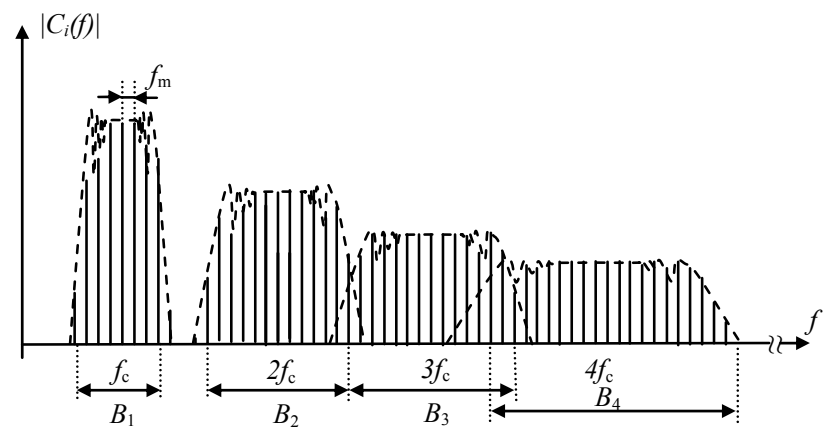

(a)

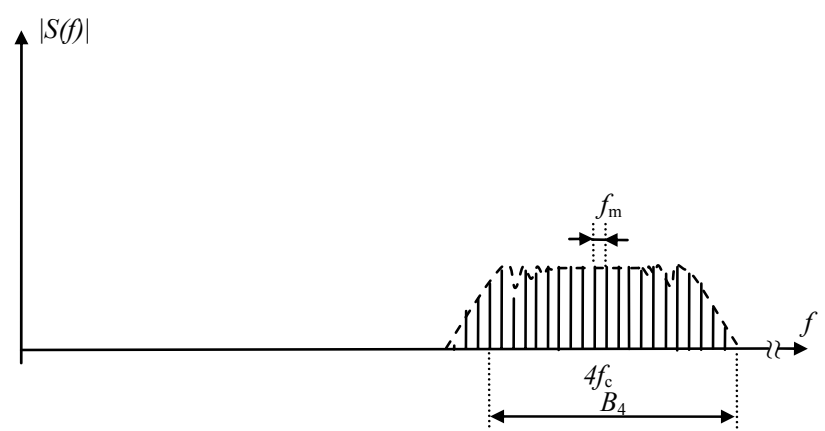

(b)

Fig. 8. Harmonics cancellation achieved with SFM with variable delay for 4 converters. (a) Switching pattern spectrum. (b) Equivalent source of noise pattern spectrum.

\section{EXPERIMENTAL SETUP}

In order to evaluate the proposed techniques, a four channel parallel buck converter has been fabricated according to the following specifications:

- Input voltage: $12 \mathrm{~V}_{\mathrm{DC}}$.

- Output voltage: $1.5 \mathrm{~V}_{\mathrm{DC}}$.

- Output power: $1 \mathrm{~W}$. 


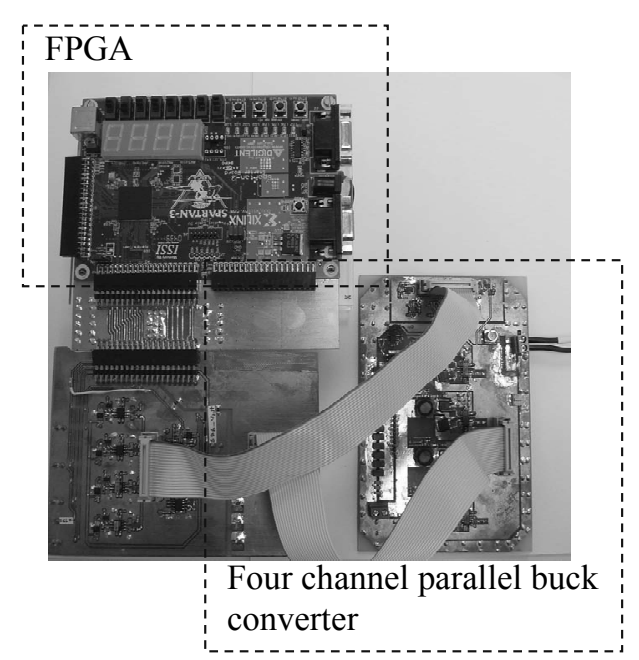

(a)

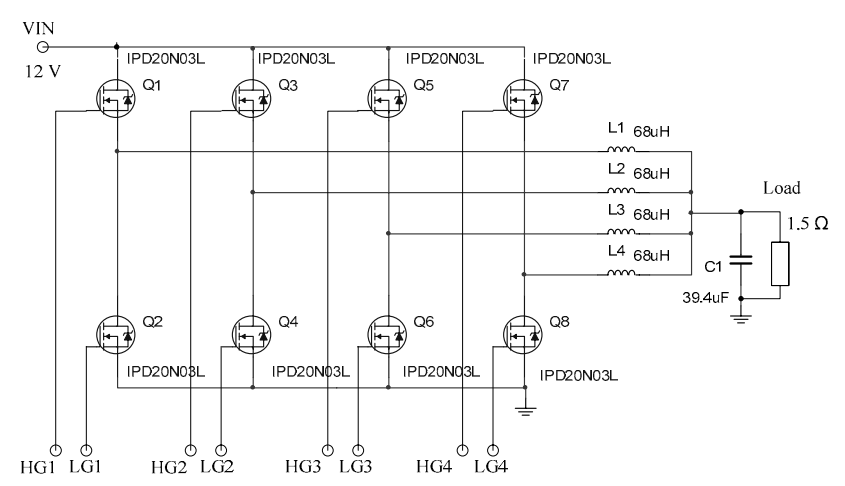

(b)

Fig. 9. Multiconverter prototype. (a) Full view prototype. (b) Schematics of four channel parallel buck converter.

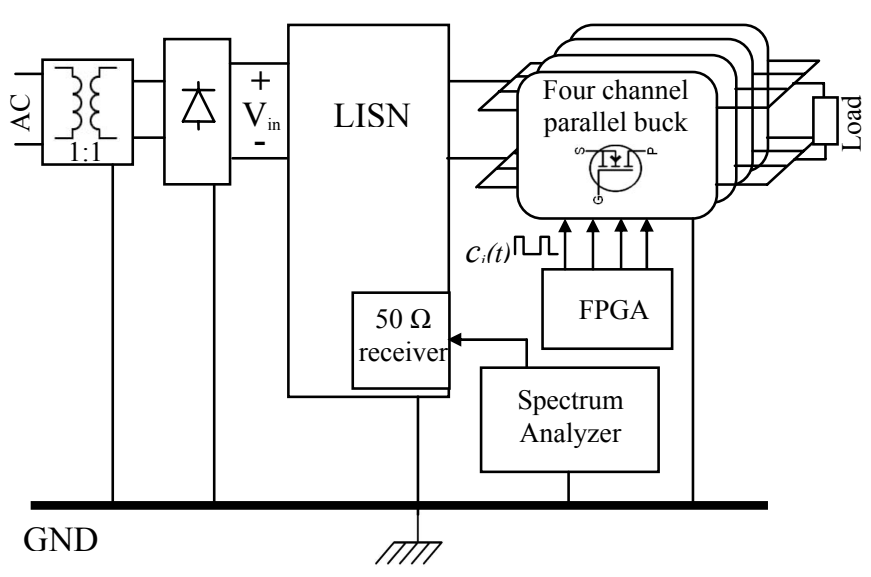

Fig. 10. Setup used in this experiment.

Figure 9 (a) and Figure 9 (b) show a picture and scheme of the multiconverter prototype, respectively.

The measurement setup is schematically depicted on Figure 10. The switching pattern has been generated by means of a field programmable gate array (FPGA), and the noise spectrum of parallel converter has been measured with a spectrum analyzer through line impedance stabilization network (LISN). The experimental results have been obtained for a duty cycle $D=12.5 \%$, central switching frequency $f_{c}=400 \mathrm{kHz}$, a sawtooth modulation profile, $V_{m}(t)$, with a modulation frequency $f_{m}=10 \mathrm{kHz}$ and a maximum frequency deviation $\Delta f_{c}=60 \mathrm{kHz}$.

\section{RESULTS}

First of all, in order to evaluate the measurement setup, the noise spectrum of parallel buck converter without any EMI reduction techniques has been compared with interleaving and frequency modulation techniques. Figure 11 shows the noise spectrum with and without interleaving techniques. A strong reduction, about $20 \mathrm{~dB}$, on all harmonics not multiple of $4 \cdot f_{c}$ is observed and only harmonics multiple of $4 \cdot f_{c}$ are not reduced, such as expected theoretically (Fig.4).

Figure 12 shows the comparison between the EMI spectrum with and without SFM technique. For SFM, distributions of harmonic energy in side-bands generated by modulation process are observed, with an amplitude reduction about $10 \mathrm{~dB}$. These results confirm the theoretically expected results (Fig.1) and moreover validate our measurement setup.

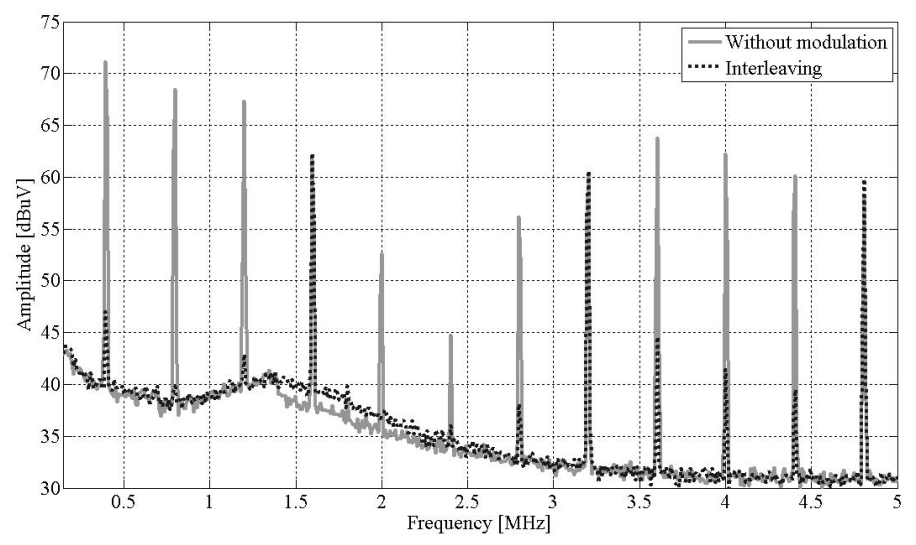

Fig. 11. EMI comparison with and without interleaving.

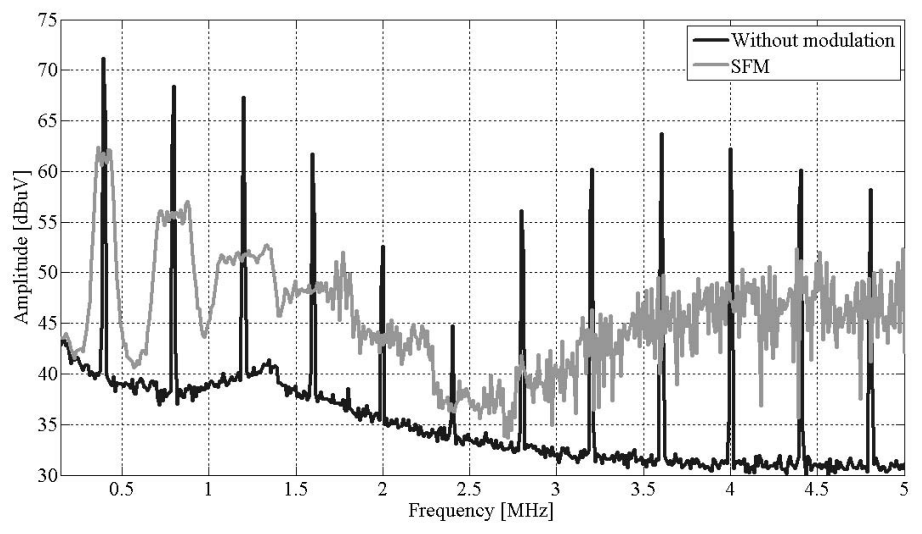

Fig. 12. EMI comparison: Without modulation vs SFM. 


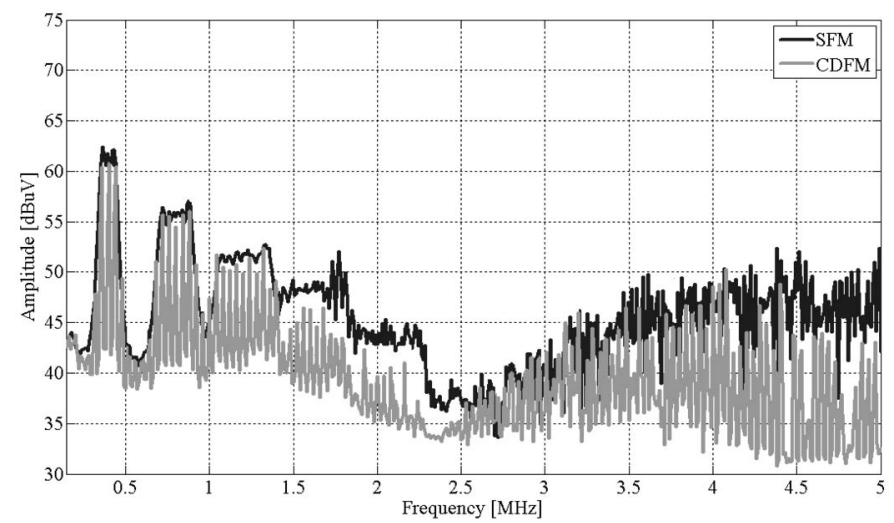

Fig. 13. EMI comparison of SFM and CDFM.

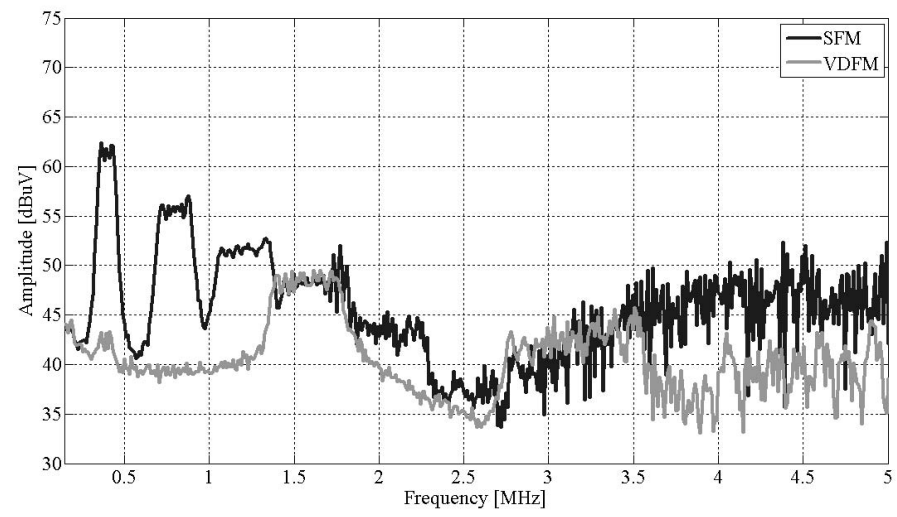

Fig. 14. EMI comparison of SFM and VDFM.

In Figure 13 SFM are compared with the first proposed techniques (CDFM) in terms of noise spectrum. In case of CDFM, the distance between two adjacent side-band harmonics increase up to $4 \cdot f_{m}$. and an amplitude reduction about $3 \mathrm{~dB}$ is observed, compared with standard SFM technique, which is predicted theoretically (Fig.6).

Figure 14 shows the comparison between the SFM and VDCM. In this case, only side-bands harmonics multiply of $4 \cdot f_{c}$. are observed. This effect produces a noticeable EMI amplitude reduction, of more than $20 \mathrm{~dB}$, in comparison with the others reduction techniques analysed.

\section{CONCLUSIONS}

In this paper, two combination techniques for multiconverters based on interleaving and frequency modulation have been presented. The first one, namely CDFM, consist on applied a constant delay on each switching pattern. The second one, namely VDFM, consist on applied interleaving in each switching cycle. Both techniques have been implemented on a four channel parallel buck converter. The results show an EMI reduction about $3 \mathrm{~dB}$ in case of CDFM and $20 \mathrm{~dB}$ for VDFM.

In future works, additional study on EMI when the duty cycle varies will be explored, in order to compare the effectiveness of proposals techniques.

\section{ACKNOWLEDGMENT}

This research is supported by Spanish Ministry of Science and Technology, MCYT, in the frame of the project TEC2007-61582-MIC.

\section{REFERENCES}

[1] Feng Lin, Dan Y. Chen, "Reduction of Power Supply EMI Emission by Switching Frequency Modulation", IEEE Trans. on Power Electronics, vol. 9 , pp. 132 - 137, January 1994.

[2] Aleksandar M. StankoviC, George C. Verghese, David J. Perreault, "Analysis and Synthesis of Randomized Modulation Schemes for Power Converters", IEEE Trans. on Power Electronics, vol. 10, pp. 680 - 693, November 1995.

[3] Tse, K.K., Henry Shu-Hung Chung, Ron Hui, S.Y., So, H.C., "A comparative study of carrier-frequency modulation techniques for conducted EMI suppression in PWM converters", IEEE Trans. on Industrial Electronics, vol. 49, pp. 618 - 627, June 2002.

[4] Balcells J., Santolaria A., Orlandi A., Gonzalez D., Gago J., "EMI reduction in switched power converters using frequency Modulation techniques", IEEE Trans. on Electromagnetic Compatibility, vol 47, pp. 569 - 576, August 2005.

[5] Johnson, S., Zane, R., "Custom spectral shaping for EMI reduction in high-frequency inverters and ballasts", IEEE Trans. on Power Electronics, vol. 20, pp. 1499 - 1505, November 2005.

[6] David González, Jan T. Bialasiewicz, Josep Balcells, Javier Gago, "Wavelet-Based Performance Evaluation of Power Converters Operating With Modulated Switching Frequency", IEEE Trans. on Industrial Electronics, vol. 55, pp. 3167 - 3176, August 2008.

[7] Luis A. Barragán, Denis Navarro, Jesús Acero, Isidro Urriza, José M. Burdío, "FPGA Implementation of a Switching Frequency Modulation Circuit for EMI Reduction in Resonant Inverters for Induction Heating Appliances", IEEE Trans. on Industrial Electronics, vol. 55, pp. 11 - 20, January 2008.

[8] C. Chang, M. Knights, "Interleaving technique in distributed power conversion systems", IEEE Trans. on Circuits and Syst. I, vol. 42, pp. 245-251, May 1995.

[9] Zumel P., Garcia O., Cobos J.A., Uceda J., "EMI reduction by interleaving of power converters", Applied Power Electronics Conference and Exposition, APEC 2004, vol. 2, pp. 688 - 694, 2004.

[10] Al-Haddad K., Rajagopalan V., "Analysis and Simulation of Multiple Converter Operation to Reduce Losses and EMI", IEEE Workshop on Computers in Power Electronics, pp. 183 - 188, August 2004.

[11] Saurabh Jayawant, Jian Sun, "Double-Integral Fourier Analysis of Interleaved Pulse Width Modulation", IEEE COMPEL Workshop, Rensselaer Polytechnic Institute, Troy, NY, USA, pp. 34 - 39, July 2006.

[12] Ozeri S., Shmilovitz D., Singer S., Martinez-Salamero L., "The Mathematical Foundation of Distributed Interleaved Systems", IEEE Trans. on Circuits and Systems--I: vol. 54, pp. 610-619, March 2007. 